\title{
E74 like ETS transcription factor 3 (ELF3) is a negative regulator of epithelial-
}

\section{mesenchymal transition in bladder carcinoma}

Kirti Gondkar ${ }^{1,2}$, Krishna Patel ${ }^{1,2}$, Shoba Krishnappa ${ }^{3}$, Akkamahadevi Patil ${ }^{4}$, Bipin Nair ${ }^{2}$, Gopinath Meenakshi Sundaram ${ }^{5}$, Tan Tuan Zea ${ }^{6}$, Prashant Kumar ${ }^{1,7^{*}}$

${ }^{1}$ Institute of Bioinformatics, International Tech Park, Bangalore, India

${ }^{2}$ Amrita School of Biotechnology, Amrita Vishwa Vidyapeetham, Kollam, India

${ }^{3}$ Department of Surgical Oncology, Kidwai Memorial Institute of Oncology, Bangalore, India

${ }^{4}$ Department of Pathology, Kidwai Memorial Institute of Oncology, Bangalore India.

${ }^{5}$ Department of Biochemistry, Central Food Technological Research Institute, Mysore, India

${ }^{6}$ Cancer Science Institute of Singapore, National University of Singapore, Singapore

${ }^{7}$ Manipal Academy of Higher Education (MAHE), Manipal, Karnataka, India

\section{CORRESPONDENCE}

Prashant Kumar, Ph.D.

Faculty Scientist

Institute of Bioinformatics

$7^{\text {th }}$ floor, Discoverer building,

International Tech Park,

Bangalore 560 066, India

E-mail: prashant@ibioinformatics.org

$\begin{array}{llll}\text { Authors } & \text { Telephone } & \text { Fax } & \text { Email } \\ \text { Kirti Gondkar } & 91-80-28416140 & 91-80-28416132 & \underline{\text { kirti@ibioinformatics.org }} \\ \text { Krishna Patel } & 91-80-28416140 & 91-80-28416132 & \underline{\text { krishnapatel@ibioinformatics.org }} \\ \text { Shoba Krishnappa } & 91-9845880095 & -- & \underline{\text { drshoba2010@ gmail.com }} \\ \text { Devi Patil } & 91-9035884253 & -- & \underline{\text { devispatil@yahoo.co.in }} \\ \text { Bipin Nair } & 91-476-2803000 & 91-476-2801280 & \underline{\text { bipin@am.amrita.edu }} \\ \text { Gopi Meenakshisundaram } & 91-9686691486 & -- & \text { gopinath@cftri.res.in } \\ \text { Tan Tuan Zea } & -- & -- & \underline{\text { csittz@nus.edu.sg }} \\ \text { Prashant Kumar } & 91-80-28416140 & 91-80-28416132 & \text { prashant@ibioinformatics.org }\end{array}$




\section{Abstract}

Transcription factors are known to be commonly deregulated in various cancers. The E74 like ETS transcription factor 3 (ELF3) expression is restricted to epithelial tissue. In the present study, we evaluated the role of ELF3 in the pathogenesis of bladder carcinoma (BCa) using cell line model. The cell lines with low expression of ELF3 showed increased expression of mesenchymal markers and decreased expression of epithelial markers. Immunofluorescence and immunohistochemical analysis of ELF3 showed selective expression in low-grade BCa cell lines and tumor tissues, respectively. We demonstrated that overexpression of ELF3 in UMUC3, a mesenchymal BCa cell line resulted in reduced invasion and decreased expression of mesenchymal markers. Furthermore, using publicly available data, we found that low expression of $E L F 3$ was associated with increased risk and poor overall survival rate in $\mathrm{BCa}$. In conclusion, ELF3-modulated reversal of EMT might be a useful strategy in the treatment of bladder cancer.

\section{Introduction}

Bladder carcinoma is incredibly heterogeneous, comprises multiple disease entities with distinct molecular features and biological characteristics.[1] Low-grade non-muscle-invasive (NMI) tumors have high recurrence frequency of $70 \%$ and $20 \%$ of them progress to muscle invasive tumors (MI).[2] Muscle-invasive tumors are generally diagnosed de novo and are involved in metastasis. MI and NMI are two distinct tumors with different clinico-pathological characteristics. NMI carcinoma develop via epithelial hyperplasia whereas MI develop via flat dysplasia and carcinoma in situ (CIS).[1] The risk of progression of NMI to MI after 5 years ranges from 6\% to 45\% [3] with less than $15 \%$ of 5 year survival rate.[4] Treatments have not advance beyond cisplatin or cisplatin containing combination chemotherapies for decades. [5] A detailed understanding of bladder carcinoma pathogenesis is an unmet need.

Various studies suggest multifocal occurrence is the characteristic feature of bladder carcinoma. At molecular levels, epithelial to mesenchymal transition (EMT) could play a major role in progression of NMI to MI.[6-9] This transition in cell phenotype is also associated with aggressiveness, recurrence, and metastases of tumors.[10] However, our understanding about the disease pathogenesis is still not evolved comprehensively. EMT is known to be governed by 
several transcription factors, such as SNAIL, SLUG, ZEB1, ZEB2, and TWIST among others, that transcriptionally repress epithelial markers.[11] These molecules are often referred as EMT transcription factors (EMT-TFs).

Among families of transcription factors, there are increasing evidence for E-twenty six (ETS) transcription factor family members to regulate EMT in cancer.[12-15] ETS family members usually expressed by epithelial cells and are largely implicated in matrix metalloproteinase (MMP) regulation.[16] ETS-related transcription factors are also involved in cell proliferation, adhesion, motility/migration, cell survival, invasion, extravasation, micro-metastasis, establishment and maintenance of distant site metastasis and angiogenesis.[15-17] Member of ETS family, ELF5 (E74 Like ETS Transcription Factor 5) is shown to directly suppress EMT by repressing the transcription of SLUG.[12] ETV1, another member of ETS family also acts to promotes snail expression to induce EMT in gastric cancer.[18] Likewise, ELF3 (E74 like ETS transcription factor 3), also a member of ETS family is restricted to epithelial tissue expression.[19, 20] Higher expression of ELF3 was reported in epithelial rich tissues such as colon, small intestine and negligible expression in epithelial poor tissues such as brain, heart, spleen, thymus and skeletal muscles.[19, 20] Furthermore, expression of ELF3 is negligible in non-epithelial origin cells like lymphocytes, monocytes, and endothelial cells.[20]

Mutation profile studies have identified recurrent anomalies in multiple genes and pathways that are potential key drivers of bladder carcinoma.[21-23] A large-scale genomics study from The Cancer Genome Atlas (TCGA) identified 58 genes as significantly mutated across 412 muscle invasive bladder carcinoma.[24] ELF3 was reported with frequency of $12 \%$ non-synonymous mutation in these tumors. However, the prognostic and functional significance of ELF3 are yet to be explored thoroughly in bladder carcinoma. Here we demonstrate the inverse correlation of ELF3 expression with EMT dynamics along with its association with overall survival. We also studied the effect of ELF3 overexpression on bladder carcinoma invasiveness using cell based invasion assay. 


\section{Methods}

\section{Cell culture}

Five bladder carcinoma cell lines UMUC3, J82, SW780, RT112, T24 were acquired from ATCC and cultured in Dulbecco's modified eagle medium supplemented with $10 \%$ fetal bovine serum, $1 \%$ sodium pyruvate and $1 \%$ penicillin- streptomycin mixture. Cell lines were grown and maintained in humidified $5 \% \mathrm{CO}_{2}$ atmosphere at $37^{\circ} \mathrm{C}$.

\section{Transient Transfection}

Routinely maintained UMUC3 cells were transfected by piRES-puro-ELF3 (Addgene cat.no. 25728) using XtremeGENE HP DNA Transfection reagent, Roche, as per manufacture instructions. For western blotting, cells were harvested 72 hours after transfection. For Invasion assay, cells were seeded 24 hours post transfection into boyden chamber.

\section{Cell invasion assay}

The invasive property of UMUC3 was measured using the BD BioCoat ${ }^{\mathrm{TM}}$ Matrigel ${ }^{\mathrm{TM}}$ Invasion guidelines. Briefly, boyden chamber inserts (Thermo Fisher Scientific, Waltham, MA, USA) were coated with $20 \mu 1$ 1:10 matrigel and allowed to solidify at room temperature for 1 hour. Cells were seeded in duplicates at $20 \times 10^{4}$ in $0.5 \mathrm{ml}$ serum free media, while the lower chamber contained $10 \%$ FBS. Cells were allowed to invade through the porous membrane coated with matrigel at $37^{\circ} \mathrm{C}$ for 48 hours. Inserts were fixed, stained and photographed in 5 fields per insert. Cell count was performed to determine relative invasion.

\section{Western blotting}

Whole cell extracts from 70-80\% confluent cells were prepared using modified RIPA lysis buffer (Merck Millipore, Billerica, MA,) containing protease inhibitors (Roche, Indianapolis, IN,) and phosphatase inhibitors (Thermo Scientific, Bremen, Germany). Western blot analysis was performed on 10\% SDS- PAGE using $30 \mu \mathrm{g}$ protein lysates. After separation proteins were transferred onto nitrocellulose membrane (BioRad) and were hybridized with primary antibodies. Pre blocking in 5\% milk for 30 mins was carried out for polyclonal antibodies. Protein bands were visualized using Luminol reagent (Santa Cruz Biotechnology, Dallas, TX,) as per the manufacturer's instructions. 


\section{Gondkar et al. 2018}

Anti ELF3 antibody was obtained from R\&D systems. E-cadherin and slug, antibodies were obtained from Cell

Signaling Technology (Cell Signaling Technology, Beverly, MA). Anti N-cadherin and GAPDH were obtained from Abcam. Anti Claudin7 was obtained from Acris antibodies.

\section{Immunohistochemistry}

Bladder carcinoma formalin fixed paraffin embedded tissue sections were obtained following institutional review board approval and informed consent from Kidwai institute of molecular oncology (KMIO/MEC/011/24.November.2016). The sections were deparaffinised and antigen retrieval was carried out using heat-induced epitope retrieval by incubating them for 20 minutes in antigen retrieval buffer ( $0.01 \mathrm{M}$ Trisodium citrate buffer, $\mathrm{pH}$ 6). The quenching of endogenous peroxidases was done by using a blocking solution followed by washes with wash buffer (PBS with $0.05 \%$ Tween-20). The sections were incubated with primary antibody overnight at $4^{\circ} \mathrm{C}$ in a humidified chamber. Anti-ELF3 antibody (R\&D systems) was used at 1:200 dilutions. After incubation with the primary antibody, the sections were washed thrice with wash buffer. The slides were incubated with appropriate horseradish peroxidase conjugated goat secondary antibody for 30 minutes at room temperature. Excess secondary antibody was removed using wash buffer followed by addition of DAB substrate. The signal was developed using DAB chromogen (DAKO, Glostrup, Denmark). The immunohistochemical labeling was assessed by an experienced pathologist.

\section{Immunofluorescent staining}

Approximately $3 \times 10^{3}$ cells were seeded in chamber vials and grown for 48 hours in humidified $5 \% \mathrm{CO}_{2}$. Cells were fixed with 4\% Paraformaldehyde (PFA) (Merk, India) and then permeabilised with 0.5\% Triton-X100 (HiMedia, India). Cells were then blocked with $0.1 \%$ Triton-X100 in 3\% BSA (HiMedia, India). Samples were stained with primary antibody ELF3 (R\&D systems), and secondary antibodies - Alexa Fluor® 568-labeled donkey anti-goat $\operatorname{IgG}$ (A11057, Invitrogen, CA, USA) and DAPI (D1306, Invitrogen, CA, USA). Images were acquired under inverted epifluorescent microscope (Olympus IX73, USA).

\section{Computational analysis}




\section{Gondkar et al. 2018.}

Normalized intensities of twenty-six ETS family members were compiled from Earl et al., 2015 for sixteen cell lines.[25] These sixteen cell lines were stratified into group of epithelial cell lines (Epi) ( $\mathrm{n}=9$ ), epithelial or mesenchymal (E/M) (n=2) and mesenchymal (Mes) $(\mathrm{n}=5)$ cell lines, as described by Tan et al., 2014.[26] The gene expression matrix was processed using Morpheus (https://software.broadinstitute.org/morpheus/) to generate heatmap with one-minus Pearson correlation clustering approach. SurvExpress database (http://bioinformatica.mty.itesm.mx:8080/Biomatec/SurvivaX.jsp) was used to obtain risk group information for bladder carcinoma.[27] All the 5 different bladder carcinoma datasets were considered for analysis. Risk groups with $p$-values $\leq 0.05$ was considered significant.

We also analyzed expression of ELF3 using web-based platforms such as UALCAN[28] for Kaplan-Meier overall survival analysis to corroborate our cell-line based findings with patient data-sets from The Cancer Genome Atlas (TCGA).

\section{Computation of EMT score}

Meta-cohort of bladder carcinoma dataset Affymetrix U133A platform was compiled in Tan et al., 2014.[26] Briefly, bladder carcinoma from GSE31684, GSE7476, and GSE5287 were downloaded from GEO. The data were RMAnormalized and combined using ComBat.[29] Clinical and processed RNA-seq data of TCGA bladder carcinoma cohort was downloaded from GDAC (version 2016_01_28) (Broad2016). The FPKM values of ELF3 were extracted for downstream analysis. 


\section{Results}

\section{Expression pattern of ELF3 and ETS family members}

EMT score computed by Tan et al., 2014 classified RT4, UMUC9, HT1197, HT1376, BC16.1, CUBIII, SCABER, UMUC6, PSI, 5637, KU7, as epithelial cell lines.[26] HU456 and KK47 were stratified as cell lines showing both epithelial and mesenchymal characteristics.[26] Whereas 253JBV, T24, TCCSUP, J82, UMUC13 and UMUC3 showed most mesenchymal characteristics. We further utilized same classification to assess expression pattern of ETS family members from microarray data provided by Earl et al., 2015 across 40 bladder carcinoma cell lines (Supplementary table S1).[25] We observed that ELF2, ELF1, ETS2, ERF, ELF4, ETV4, ETS1, SPDEF, ELK1, FEV, ETV1 were highly expressed genes across cell lines. Whereas FL1, ELK4, ETV3, ERG and ETV2 showed relatively low expression across cell lines (Figure 1A). We observed ELF3 expression was relatively low in cell lines characterized as mesenchymal (T24, TCCSUP, J82, UMUC13 and UMUC3) and high in cell lines characterized as epithelial (RT4, UMUC9, HT1197, HT1376, PSI and 5637) (Figure 1B). Other epithelial cell lines SCABER and UMUC6 presented moderate expression of ELF3 whereas UMUC14 exhibited low expression of ELF3. ELF3 expression difference was assessed using non-parametric unpaired Mann-Whitney test and we observe that it was significantly higher in epithelial cell lines compared to mesenchymal cell lines with $p$-value of 0.0280 .

A

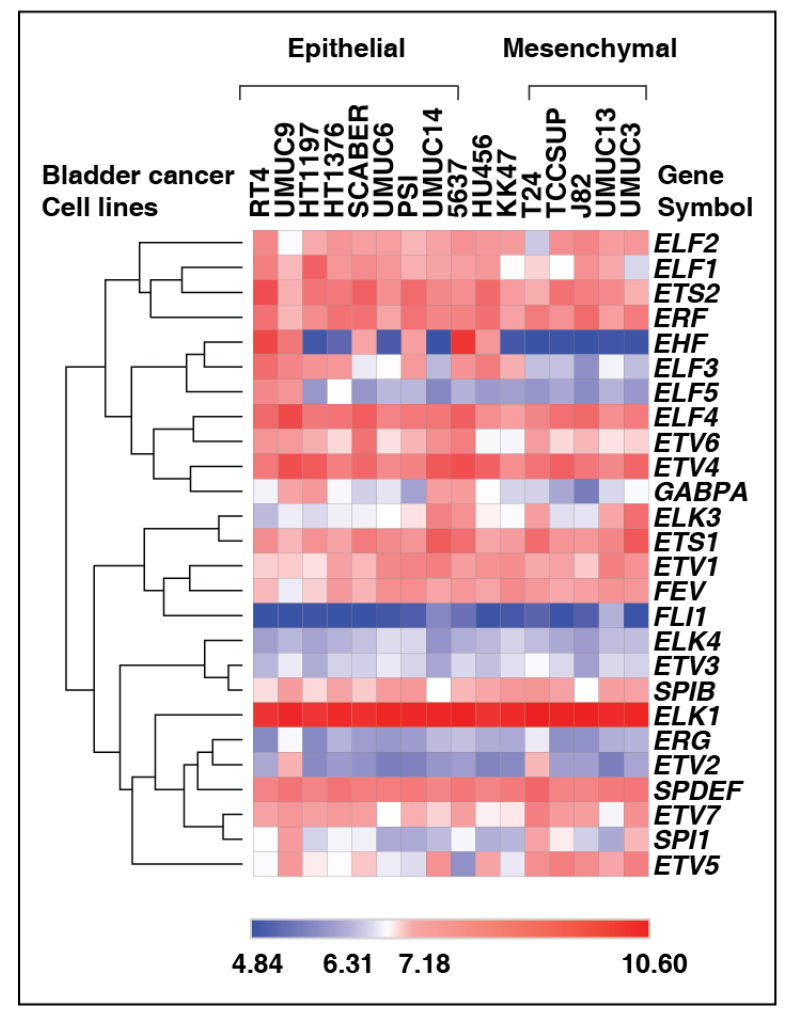

\section{B}

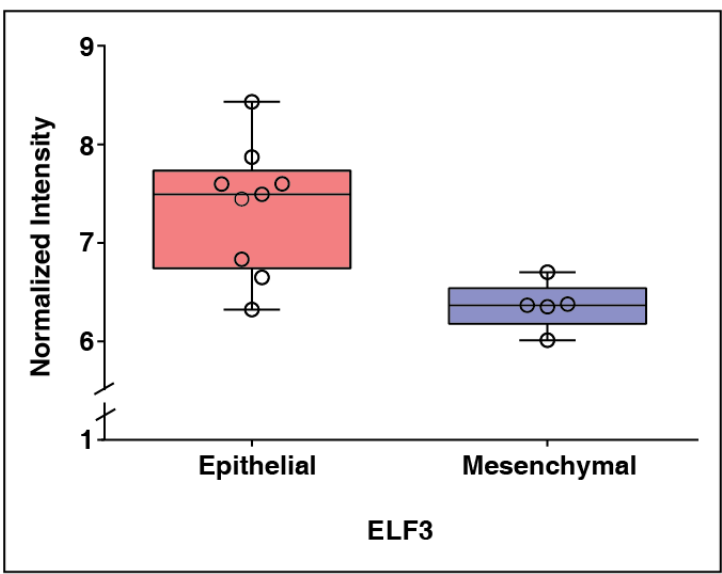

Figure 1. Expression profile of ETS family in bladder carcinoma cell lines. (A) Heatmap representing normalized intensities of ETS family from epithelial (RT4, UMUC9, HT1197, HT1376, BC16.1, CUBIII, SCABER, UMUC6, PSI, 5637, KU7), mixed (HU456 and KK47) and mesenchymal (253JBV, T24, TCCSUP, J82, UMUC13, UMUC3) tumor derived cell lines reported using microarray analysis (B) Relatively low expression of ELF3 in mesenchymal compared to epithelial tumor derived cell lines 


\section{Gondkar et al. 2018.}

\section{ELF3 is down regulated in cell lines derived from high grade tumors}

To confirm the expression pattern of ELF3, western blot was performed across 5 bladder carcinoma cell lines. These cell lines were established from low-grade tumor to high-grade tumors (Supplementary table S2). As shown in figure 2A-E and figure 3A, cell lines derived from low grade tumor, SW780 and RT112 expressed ELF3 while other cell lines T24 and J82 derived from high grade tumors and also characterized as most mesenchymal cell lines along with UMUC3 showed negligible expression of ELF3. We also observed varied localization pattern of ELF3 with respect to grade of the cell lines. ELF3 was localized in nucleus and cytoplasm in SW780 and RT112 whereas in T24 and J82, ELF3 was localized in negligible amounts in cytoplasm and absent in nucleus.

Figure 2

A

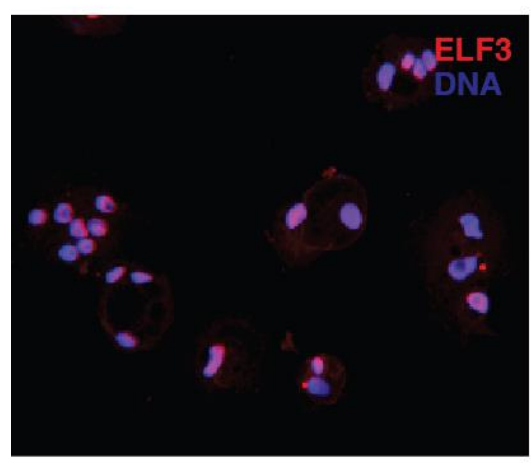

sW780

D

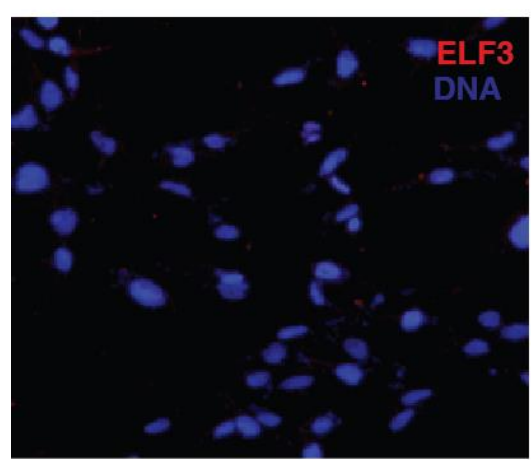

J82
B

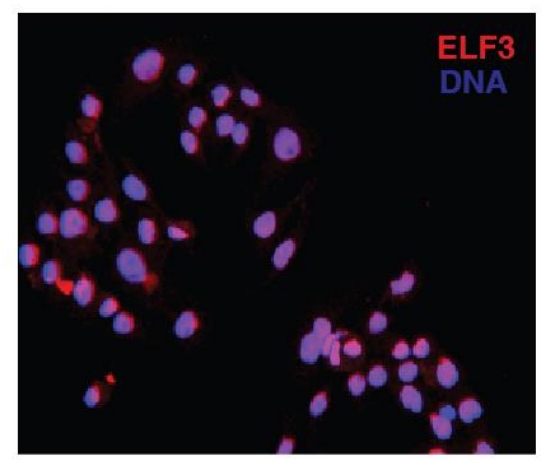

RT112

E

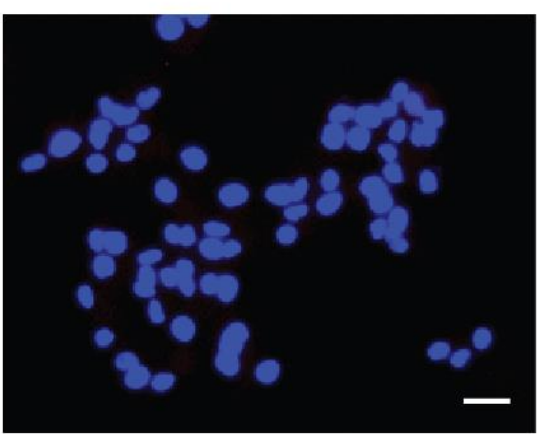

Negative control
C

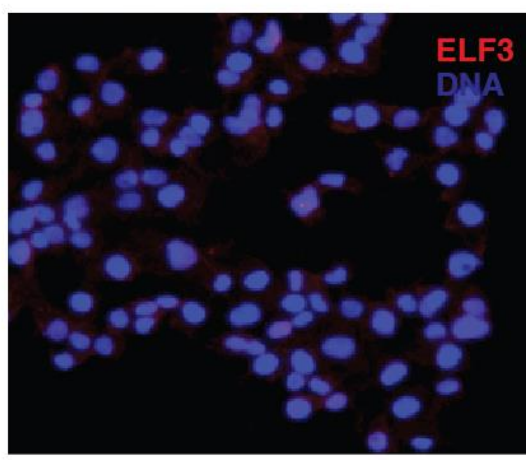

T24

Figure 2. Subcellular localization of ELF3 in bladder carcinoma cell lines. Immunofluorescence staining showed cytoplasmic and nuclear localization of ELF3 in (A) SW780 (B) RT112; and cytoplasmic in (C) T24, (D) J82. (E) Negative control 


\section{ELF3 expression is associated with epithelial to mesenchymal transition in bladder carcinoma cells}

To study the functional role of ELF3 in EMT process, western blot analysis was performed in bladder carcinoma cell lines against the expression pattern of various EMT markers. We observed low expression of ELF3 correlated with low expression of epithelial markers E-cadherin and Claudin7 in high grade bladder carcinoma cell lines, J82, T24 and mesenchymal cell line UMUC3. Whereas expression of mesenchymal markers such as N-cadherin and SLUG were higher in high grade compared to low grade bladder carcinoma cell lines (Figure 3A). Expression of ELF3 might be involved in negative regulation of EMT in bladder carcinoma. To further validate our findings, we compared expression of ELF3 with the generic EMT signature derived from bladder carcinoma patient data sets as described by Tan et al., 2014.[26] This scoring method was developed to quantitatively estimate the EMT phenotype across clinical samples as well as cell lines using transcriptomics. Based on this in-silico analysis, we identified that low $E L F 3$ expression significantly correlated $($ Rho $=-0.7645)$ with higher mesenchymal phenotype in bladder carcinoma patients (Figure 3B).

\section{Figure 3}

A

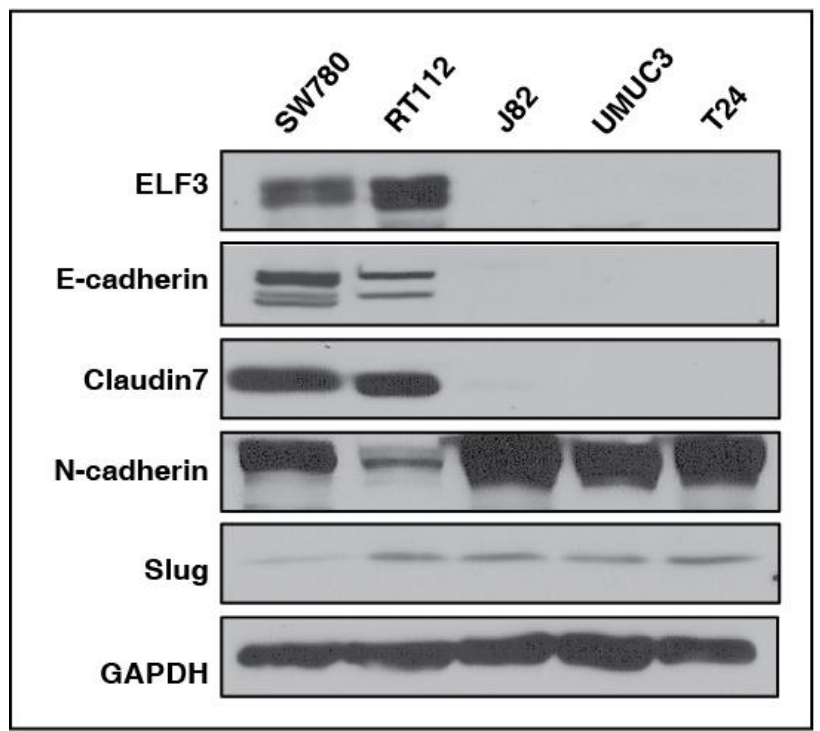

B

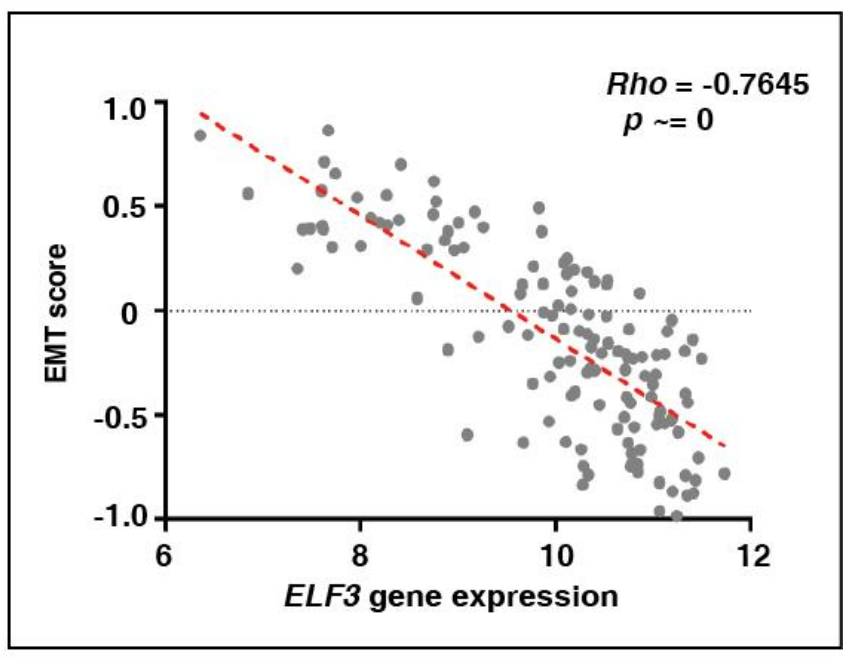

Figure 3. Association of ELF3 expression with EMT signature (A) Western blot analysis depicting higher expression of epithelial markers E-cadherin and Claudin7 in SW780 and RT112; and higher expression of mesenchymal markers N-cadherin and slug in J82, UMUC3 and T24 (B) Spearman's inverse correlation of EMT signature with ELF3 expression in bladder carcinoma patient data procured from Tan et al. 
Over expression of ELF3 decreases invasion in mesenchymal bladder carcinoma cell line UMUC3

Expression of ELF3 was downregulated across high-grade bladder carcinoma cell lines. We further sought to identify whether ELF3 overexpression can potentially revert the invasiveness in UMUC3 cell line. We observed significant decreased invasiveness of UMUC3 (Figure 4A). ELF3 over expression also showed marginal decrease in expression of mesenchymal markers like N-cadherin and SLUG, however there was no change in the expression of epithelial markers (Figure 4B) as confirmed by western blot.

\section{A}

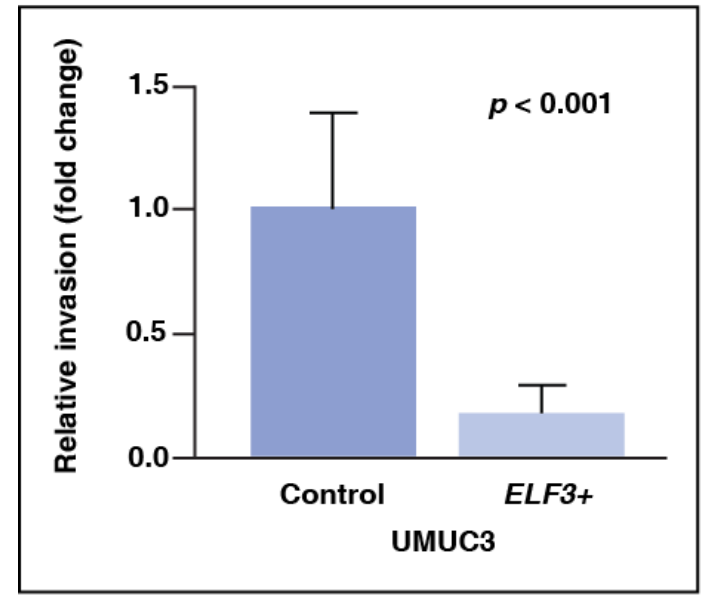

C

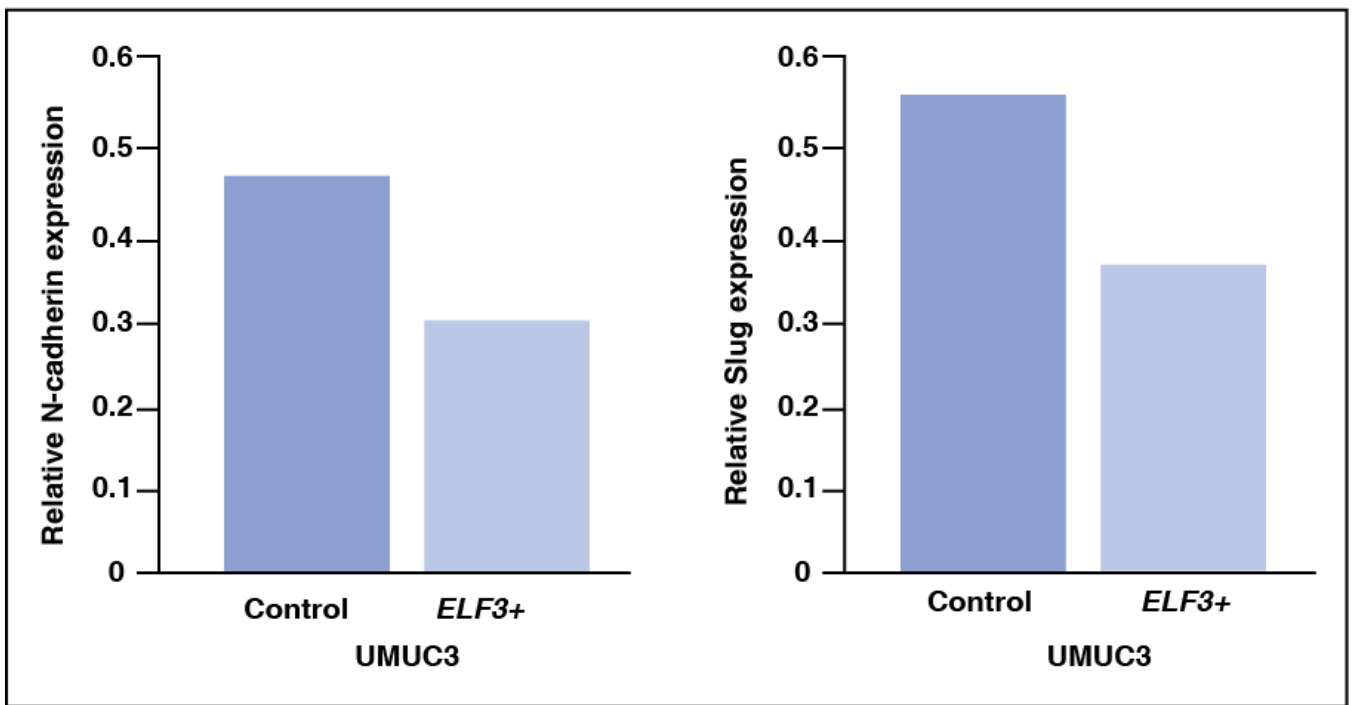

Figure 4. Overexpression of ELF3 reduces cell invasion in-vitro (A) Transient transfection of ELF3 in UMUC3 lead to decreased invasiveness $(\mathbf{B}, \mathbf{C})$ Western blot and imageJ analysis indicates lower expression of mesenchymal markers and unchanged expression of epithelial markers. 


\section{Immunohistochemistry revealed higher expression of ELF3 in low grade compared to high grade tumors}

To further investigate the expression pattern of ELF3, we performed immunohistochemical staining on bladder tumors. Patient presented with low grade carcinoma showed unchanged ELF3 expression in tumor compared to adjacent normal (Figure 5A, B). Whereas patient presented with high grade carcinoma showed decreased level of ELF3 expression in tumor compared to adjacent normal (Figure 5C, D). We observed considerable difference in the ELF3 expression between low grade and high grade tumors. In addition, we also observed decreased ELF3 expression in mesenchymal cells compared to the epithelial cells in the high grade tumor (Figure 5E). Our result suggests that ELF3 expression is higher in low grade compared to high grade tumors.

A

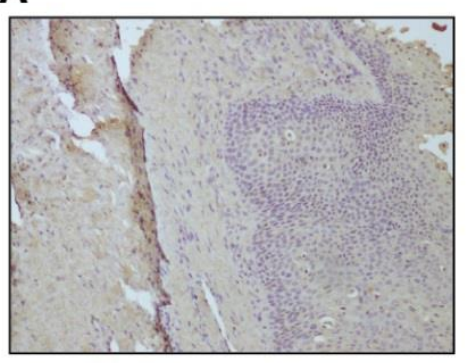

Adjacent Normal

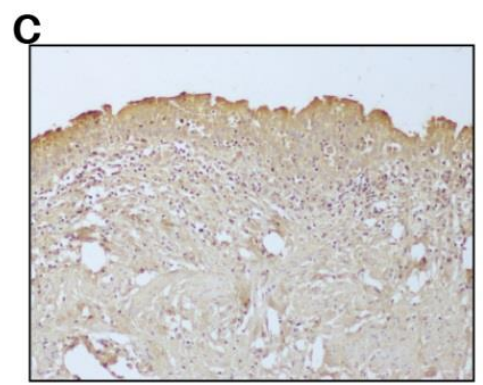

Adjacent Normal
B

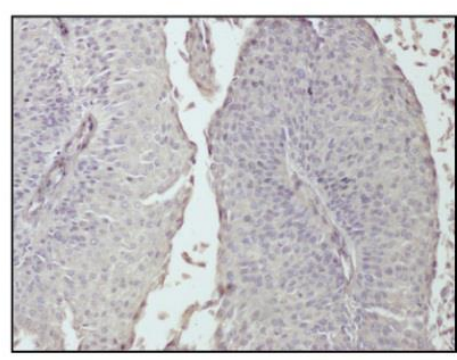

Low grade

D

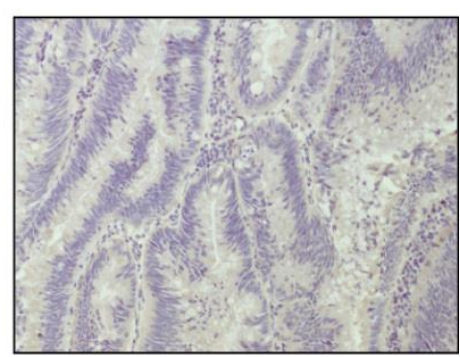

High grade

Figure 5

E

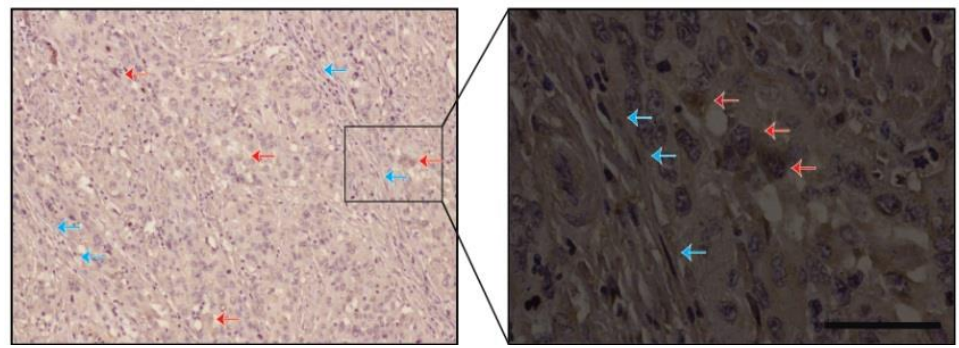

Figure 5. ELF3 expression in different grades of urothelial tumors. Immunohistochemical staining show comparable expression of ELF3 in (A) adjacent normal and (B) tumor of low grade urothelial carcinoma; whereas decreased expression from (C) adjacent normal to (D) tumor of high grade urothelial carcinoma. (E) IHC image depicts decreased expression of ELF3 in mesenchymal cells compared to epithelial cells (Blue arrows: mesenchymal cells. Red arrows: epithelial cells) 


\section{Association of ELF3 downregulation with high risk and overall poor survival in bladder carcinoma}

To assess potential risk associated with ELF3 expression in bladder carcinoma we have used five published studies hosted on an online biomarker validation tool SurvExpress.[27] These datasets were classified into low risk and high risk groups, and $p$-value was calculated using student $t$ test. From the aforementioned five data-sets, four i.e. bladder urothelial carcinoma TCGA ( $\mathrm{n}=54)$, GSE31684 ( $\mathrm{n}=93)$, GSE5287 $(\mathrm{n}=30)$ and BLCA-TCGA-Bladder urothelial carcinoma (n=390) presented that low gene expression of ELF3 was significantly correlated to high risk with hazard ratios of $1.28,1.08,0.87,1.5$ respectively (Supplementary figure 1)

To investigate the association between ELF3 expression and overall survival, we analysed publicly available datasets using Kaplan-Meier method using UALCAN.[28] Survival analysis revealed patients with low ELF3 expression $(\mathrm{n}=304)$ had poor overall survival compared to patients with high expression $(\mathrm{n}=102, \log$ rank $p$ value $=0.12)$ (Supplementary figure 2). Results suggest that low expression of ELF3 is correlated with adverse outcomes.

\section{Discussion}

ELF3 also named as ESX, ERT and ESE-1 is an epithelial-restricted member of the ETS transcription factor family, and involved in wide range of cellular processes such as differentiation, and inflammatory response.[28, 30] It is one of the critical regulators involved in urothelial cyto-differentiation in normal human urothelial (NHU) cell line.[30] Expression of ELF3 is reported in nucleus as well as in cytoplasm.[31] ELF3 positively regulates expression of TGFßR-II[32] and is critically important in regulation of intestinal epithelial differentiation in mice.[33] Mounting evidence suggests that transcription factors play a key role in cancer progression.[34] Like most transcription factors, ETS proteins act as both oncogene and tumor suppressors.[35] It has been also reported that ELF3 controls the transcription of various genes that are involved in extracellular matrix remodeling like collagenase-1, matrix metalloproteinases MMP1 and MMP13, further enhancing the process of cartilage remodeling or degradation and tumorigenesis. [36, 37]

We interrogated ELF3 expression in TCGA datasets using web-based resource UALCAN to corroborate our findings from cell line in clinical samples. UALCAN is implemented in PERL-CGI to enable web-based gene expression analysis in various stages and molecular subtypes of 31 different cancers.[28] We observed there is lower expression of ELF3 in basal/squamous ( $\mathrm{n}=142)$ bladder carcinoma subtype compared to luminal $(\mathrm{n}=246)$ and normal tissues 


\section{Gondkar et al. 2018}

(Supplementary figure 3). Squamous differentiated urothelial carcinoma are associated with advanced stages of carcinoma[38] and basal like urothelial carcinoma are characterized as non-papillary aggressive tumors with higher rates of lymph node and distant metastases compared to non-basal like urothelial carcinoma.[39]

To further understand the role of ELF3 in EMT, we investigated the expression of ELF3 in bladder carcinoma cell lines. We observed the level of ELF3 was specifically low in mesenchymal cells. Immunohistochemical analysis in low grade and high grade bladder tumors suggests decrease in the expression of ELF3 from low grade to high grade. In addition, the expression of epithelial markers were observed low in high grade cell lines and inversely correlated with the expression of mesenchymal markers. It suggests that ELF3 might be involved in regulation of EMT. In this study, we identified similar expression pattern of ELF3 and Claudin7 as reported by Kohno et al., which suggests ELF3 is indeed involved in the formation of epithelial structure in bladder cancer.[40]

Various studies have indicated localization of ELF3 affects its function. ELF3 mediates the translocation of snail and $\beta$ - catenin to cytoplasm to induce EMT.[31],[41] ELF3 is also shown to induce anchorage-independent growth and transformation of MCF-12A cell line via cytoplasmic mechanism.[42] In our study, we observed that ELF3 was specifically localized in cytoplasm in high grade bladder carcinoma cell lines T24 and J82 which might be a mechanism that imparts mesenchymal nature to these cell lines. We also observed the cytoplasmic expression of ELF3 in low-grade tumor sections. However, the exact reason for difference in localization in bladder carcinoma cell lines is not known yet.

We further sought to understand the process of bladder carcinoma progression. We checked the gain of function of ELF3 in bladder cell lines where negligible expression of ELF3 was observed. We found that upon ELF3 transfection in mesenchymal cell line UMUC3, the invasive ability of the cell line was decreased significantly. Transient transfection also showed a slight decrease in expression of mesenchymal markers N-cadherin and Slug. Subsequently we also checked the effect of ELF3 overexpression and EMT modulation in J82 and T24; however, these cell lines were very difficult to transfect and we could not get more than $10 \%$ transfection efficiency.

We observed that low expression of ELF3 correlated significantly with poor survival in bladder carcinoma patients. Our meta-analysis also suggested that patients are at higher risk with low ELF3 expression in 4 out of 5 bladder carcinoma published data-sets as per SurvExpress.[27] Low expression of ELF3 is also reported to have effect on 


\section{Gondkar et al. 2018.}

patient's overall survival and ELF3 over expression could also be used to develop novel therapeutic regimens and could be implicated to improve bladder carcinoma patient survival. Our study delineates the molecular mechanism of ELF3 mediated EMT in aggressive bladder tumors.

\section{Conclusions}

Epithelial to mesenchymal transition phenomenon majorly contributes to metastasis and cancer progression, however the molecular mechanisms underlying this transition remains to be fully understood. Our study revealed the role of ELF3 in the cellular plasticity of bladder carcinoma. This study for the first time demonstrates that expression of ELF3 negatively correlates with expression of epithelial to mesenchymal transition markers and ELF3-modulated reversal of EMT can be a valuable strategy in treatment of bladder carcinoma. 


\section{Acknowledgements}

PK is a recipient of the Ramanujan Fellowship awarded by Department of Science and Technology (DST),

Government of India. KG is a recipient of Senior Research Fellowship from University Grants Commission (UGC),

Government of India. KP is a recipient of Senior Research Fellowship from Council of Scientific Industrial Research,

Government of India.

\section{References}

[1] Knowles MA, Hurst CD. Molecular biology of bladder cancer: new insights into pathogenesis and clinical diversity. Nature reviews Cancer. 2015;15:25-41.

[2] Lelo A, Prip F, Harris BT, Solomon D, Berry DL, Chaldekas K, et al. STAG2 Is a Biomarker for Prediction of Recurrence and Progression in Papillary Non-Muscle-Invasive Bladder Cancer. Clinical cancer research : an official journal of the American Association for Cancer Research. 2018.

[3] Sylvester RJ, van der Meijden AP, Oosterlinck W, Witjes JA, Bouffioux C, Denis L, et al. Predicting recurrence and progression in individual patients with stage Ta T1 bladder cancer using EORTC risk tables: a combined analysis of 2596 patients from seven EORTC trials. European urology. 2006;49:466-5; discussion 75-7.

[4] Lobo N, Mount C, Omar K, Nair R, Thurairaja R, Khan MS. Landmarks in the treatment of muscle-invasive bladder cancer. Nature reviews Urology. 2017;14:565-74.

[5] Alfred Witjes J, Lebret T, Comperat EM, Cowan NC, De Santis M, Bruins HM, et al. Updated 2016 EAU Guidelines on Muscle-invasive and Metastatic Bladder Cancer. European urology. 2017;71:462-75.

[6] McConkey DJ, Choi W, Marquis L, Martin F, Williams MB, Shah J, et al. Role of epithelial-tomesenchymal transition (EMT) in drug sensitivity and metastasis in bladder cancer. Cancer metastasis reviews. 2009;28:335-44.

[7] Chaffer CL, Brennan JP, Slavin JL, Blick T, Thompson EW, Williams ED. Mesenchymal-to-epithelial transition facilitates bladder cancer metastasis: role of fibroblast growth factor receptor-2. Cancer research. 2006;66:11271-8.

[8] Baumgart E, Cohen MS, Silva Neto B, Jacobs MA, Wotkowicz C, Rieger-Christ KM, et al. Identification and prognostic significance of an epithelial-mesenchymal transition expression profile in human bladder tumors. Clinical cancer research : an official journal of the American Association for Cancer Research. 2007; 13:1685-94.

[9] Singh R, Ansari JA, Maurya N, Mandhani A, Agrawal V, Garg M. Epithelial-To-Mesenchymal Transition and Its Correlation With Clinicopathologic Features in Patients With Urothelial Carcinoma of the Bladder. Clinical genitourinary cancer. 2017;15:e187-e97.

[10] Yun SJ, Kim WJ. Role of the epithelial-mesenchymal transition in bladder cancer: from prognosis to therapeutic target. Korean journal of urology. 2013;54:645-50.

[11] Goossens S, Vandamme N, Van Vlierberghe P, Berx G. EMT transcription factors in cancer development re-evaluated: Beyond EMT and MET. Biochimica et biophysica acta. 2017;1868:584-91.

[12] Chakrabarti R, Hwang J, Andres Blanco M, Wei Y, Lukacisin M, Romano RA, et al. Elf5 inhibits the epithelial-mesenchymal transition in mammary gland development and breast cancer metastasis by transcriptionally repressing Snail2. Nature cell biology. 2012;14:1212-22.

[13] Colas E, Muinelo-Romay L, Alonso-Alconada L, Llaurado M, Monge M, Barbazan J, et al. ETV5 cooperates with LPP as a sensor of extracellular signals and promotes EMT in endometrial carcinomas. Oncogene. 2012;31:4778-88.

[14] Llaurado M, Abal M, Castellvi J, Cabrera S, Gil-Moreno A, Perez-Benavente A, et al. ETV5 transcription factor is overexpressed in ovarian cancer and regulates cell adhesion in ovarian cancer cells. International journal of cancer. 2012;130:1532-43. 
[15] Sizemore GM, Pitarresi JR, Balakrishnan S, Ostrowski MC. The ETS family of oncogenic transcription factors in solid tumours. Nature reviews Cancer. 2017;17:337-51.

[16] Singh S, Barrett J, Sakata K, Tozer RG, Singh G. ETS proteins and MMPs: partners in invasion and metastasis. Current drug targets. 2002;3:359-67.

[17] Hsu T, Trojanowska M, Watson DK. Ets proteins in biological control and cancer. Journal of cellular biochemistry. 2004;91:896-903.

[18] Li Z, Zhang L, Ma Z, Yang M, Tang J, Fu Y, et al. ETV1 induces epithelial to mesenchymal transition in human gastric cancer cells through the upregulation of Snail expression. Oncology reports. 2013;30:2859-63.

[19] Tymms MJ, Ng AY, Thomas RS, Schutte BC, Zhou J, Eyre HJ, et al. A novel epithelial-expressed ETS gene, ELF3: human and murine cDNA sequences, murine genomic organization, human mapping to 1q32.2 and expression in tissues and cancer. Oncogene. 1997;15:2449-62.

[20] Oettgen P, Alani RM, Barcinski MA, Brown L, Akbarali Y, Boltax J, et al. Isolation and characterization of a novel epithelium-specific transcription factor, ESE-1, a member of the ets family. Molecular and cellular biology. 1997;17:4419-33.

[21] Fujimoto K, Yamada Y, Okajima E, Kakizoe T, Sasaki H, Sugimura T, et al. Frequent association of p53 gene mutation in invasive bladder cancer. Cancer research. 1992;52:1393-8.

[22] van Rhijn BW, Lurkin I, Radvanyi F, Kirkels WJ, van der Kwast TH, Zwarthoff EC. The fibroblast growth factor receptor 3 (FGFR3) mutation is a strong indicator of superficial bladder cancer with low recurrence rate. Cancer research. 2001;61:1265-8.

[23] Orlow I, Lacombe L, Hannon GJ, Serrano M, Pellicer I, Dalbagni G, et al. Deletion of the p16 and p15 genes in human bladder tumors. Journal of the National Cancer Institute. 1995;87:1524-9.

[24] Robertson AG, Kim J, Al-Ahmadie H, Bellmunt J, Guo G, Cherniack AD, et al. Comprehensive Molecular Characterization of Muscle-Invasive Bladder Cancer. Cell. 2017;171:540-56 e25.

[25] Earl J, Rico D, Carrillo-de-Santa-Pau E, Rodriguez-Santiago B, Mendez-Pertuz M, Auer H, et al. The UBC-40 Urothelial Bladder Cancer cell line index: a genomic resource for functional studies. BMC genomics. 2015;16:403.

[26] Tan TZ, Miow QH, Miki Y, Noda T, Mori S, Huang RY, et al. Epithelial-mesenchymal transition spectrum quantification and its efficacy in deciphering survival and drug responses of cancer patients. EMBO molecular medicine. 2014;6:1279-93.

[27] Aguirre-Gamboa R, Gomez-Rueda H, Martinez-Ledesma E, Martinez-Torteya A, Chacolla-Huaringa R, Rodriguez-Barrientos A, et al. SurvExpress: an online biomarker validation tool and database for cancer gene expression data using survival analysis. PloS one. 2013;8:e74250.

[28] Chandrashekar DS, Bashel B, Balasubramanya SAH, Creighton CJ, Ponce-Rodriguez I, Chakravarthi B, et al. UALCAN: A Portal for Facilitating Tumor Subgroup Gene Expression and Survival Analyses. Neoplasia. 2017;19:649-58.

[29] Johnson WE, Li C, Rabinovic A. Adjusting batch effects in microarray expression data using empirical Bayes methods. Biostatistics. 2007;8:118-27.

[30] Bock M, Hinley J, Schmitt C, Wahlicht T, Kramer S, Southgate J. Identification of ELF3 as an early transcriptional regulator of human urothelium. Developmental biology. 2014;386:321-30.

[31] Yang X, Lee SH. Identification of ESE1 as a beta-Catenin Binding Protein. Anticancer research. 2016;36:2697-703.

[32] Choi SG, Yi Y, Kim YS, Kato M, Chang J, Chung HW, et al. A novel ets-related transcription factor, ERT/ESX/ESE-1, regulates expression of the transforming growth factor-beta type II receptor. The Journal of biological chemistry. 1998;273:110-7.

[33] Flentjar N, Chu PY, Ng AY, Johnstone CN, Heath JK, Ernst M, et al. TGF-betaRII rescues development of small intestinal epithelial cells in Elf3-deficient mice. Gastroenterology. 2007;132:1410-9.

[34] Bhagwat AS, Vakoc CR. Targeting Transcription Factors in Cancer. Trends in cancer. 2015;1:53-65.

[35] Turner DP, Watson DK. ETS transcription factors: oncogenes and tumor suppressor genes as therapeutic targets for prostate cancer. Expert review of anticancer therapy. 2008;8:33-42.

[36] Otero M, Plumb DA, Tsuchimochi K, Dragomir CL, Hashimoto K, Peng H, et al. E74-like factor 3 (ELF3) impacts on matrix metalloproteinase 13 (MMP13) transcriptional control in articular chondrocytes under proinflammatory stress. The Journal of biological chemistry. 2012;287:3559-72.

[37] Eckel KL, Tentler JJ, Cappetta GJ, Diamond SE, Gutierrez-Hartmann A. The epithelial-specific ETS transcription factor ESX/ESE-1/Elf-3 modulates breast cancer-associated gene expression. DNA and cell biology. 2003;22:79-94. 


\section{Gondkar et al. 2018}

[38] Liu Y, Bui MM, Xu B. Urothelial Carcinoma With Squamous Differentiation Is Associated With High Tumor Stage and Pelvic Lymph-Node Metastasis. Cancer control : journal of the Moffitt Cancer Center. 2017;24:78-82.

[39] Mai KT, Truong LD, Ball CG, Williams P, Flood TA, Belanger EC. Invasive urothelial carcinoma exhibiting basal cell immunohistochemical markers: A variant of urothelial carcinoma associated with aggressive features. Pathology, research and practice. 2015;211:610-8.

[40] Kohno Y, Okamoto T, Ishibe T, Nagayama S, Shima Y, Nishijo K, et al. Expression of claudin7 is tightly associated with epithelial structures in synovial sarcomas and regulated by an Ets family transcription factor, ELF3. The Journal of biological chemistry. 2006;281:38941-50.

[41] Yeung TL, Leung CS, Wong KK, Gutierrez-Hartmann A, Kwong J, Gershenson DM, et al. ELF3 is a negative regulator of epithelial-mesenchymal transition in ovarian cancer cells. Oncotarget. 2017;8:16951-63.

[42] Prescott JD, Koto KS, Singh M, Gutierrez-Hartmann A. The ETS transcription factor ESE-1 transforms MCF-12A human mammary epithelial cells via a novel cytoplasmic mechanism. Molecular and cellular biology. 2004;24:5548-64. 


\section{Supplementary Figure Legends}

Supplementary Figure 1. Association of ELF3 expression with risk groups in bladder carcinoma Downregulation of ELF3 is observed to have significant association with high risk in datasets (A) Bladder urothelial carcinoma TCGA (B) GSE31684 (C) GSE5287 (D) BLCA-TCGA, 2016 and low risk in (E) GSE13507

Supplementary Figure 2. Low expression of ELF3 is associated with overall poor survival in bladder carcinoma patients

Supplementary Figure 3. Relative low expression of ELF3 in basal squamous subtype compared to other subtypes of bladder carcinoma

\section{Supplementary Table Legends}

Supplementary Table S1: Gene expression of ETS family members across bladder carcinoma cell lines from Earl et al., 2015

Supplementary Table S2: Bladder carcinoma cell lines and their origin 